\section{THU0493 A MULTICENTRE RANDOMISED CONTROLLED FOLLOW-UP STUDY OF EFFECTS OF THE UNDERWATER TRACTION THERAPY IN CHRONIC LOW BACK PAIN}

Tamas Gati ${ }^{1}$, Agota Kulisch², Éva Czímer ${ }^{3}$, Györgyi Cserhátit ${ }^{4}$, Judit Fehér ${ }^{5}$, Mihály Oláh ${ }^{5}$, Zsuzsanna Mándó ${ }^{2}$, Tamas Bender ${ }^{1} .{ }^{1}$ Polyclinic of The Hospitaller Brothers of St John of God, Budapest, Hungary, ${ }^{2}$ St. Andrew Hospital for Rheumatic Diseases, Hévíz, Hungary; ${ }^{3}$ Sóstógyógyfürdó, Nyíregyháza, Hungary, ${ }^{4}$ Kenézy Gyula Hospital and Clinic (Debrecen), Debrecen, Hungary; ${ }^{5}$ Hungarospa Hajdúszoboszló Private Limited Company, Hajdúszoboszló, Hungary

Background: Chronic low back pain established for more than 3 months is one of the most common problems in the world. The prevalence could reach the $33 \%$.

Objectives: To investigate the effects of underwater traction therapy on chronic low back pain.

The primary objective was to prove the hypothesis that underwater traction therapy has favourable effect of LBP using the change in the clinical parameters. Our secondary objective was to evaluate whether it also leads to the improvement in the quality of life.

Methods: A prospective, multicenter, comparative (intervention arm vs. control arm), randomized follow-up study.

Participants aged between 18 and 85 years with more than 3 months low back pain and selected from outpatient clinics.

The participants were randomized to three groups: underwater weight bath traction therapy, weight bath and non-steroidal anti-inflammatory drugs (NSAIDs) medication and only non-steroidal anti-inflammatory drugs (NSAIDs) medication.

During the traction therapy ankle weights were used.

The following parameters were measured before, right after, and nine weeks after the three-week therapy: level of low back pain in rest, level during activity tested using the Visual Analog Scale (VAS); specific questionnaire on back pain (Oswerty); questionnaire on quality of life (EuroQual-5D) and clinical parameters.

Results: 141 participants aged $57.67( \pm 13.04)$ years. All of the investigated parameters improved significantly $(p<0.001)$ in the underwater weight bath traction therapy groups by the end of the treatment compared to the base period, and this improvement was persistent during the follow-up period. There were no significant changes in the measured parameters in the control group except for the Oswestry Disability Index, which may also be the result of that group receiving pain-relieving drug therapy.

Conclusion: Based on our results, underwater weight bath traction therapy, might have favourable impact on the clinical parameters and quality of life of patients suffering from chronic low back pain.

\section{REFERENCES:}

[1] Alrwaily M, Almutiri M, Schneider M. Assessment of variability in traction interventions for patients with low back pain: a systematic review. Chiropr Man Therap. 2018 Sep 17;26:35. doi: 10.1186/s12998-018-0205-z. eCollection 2018. Review. PubMed PMID: 30237870; PubMed Central PMCID: PMC6139896.

[2] Kurutz M, Oroszváry L. Finite element analysis of weightbath hydrotraction treatment of degenerated lumbar spine segments in elastic phase. J Biomech. 2010 Feb 10;43(3):433-41. doi: 10.1016/j.jbiomech.2009.10.004. Epub 2009 Nov 1. PubMed PMID: 19883918.

[3] Oláh M, Molnár L, Dobai J, Oláh C, Fehér J, Bender T. The effects of weightbath traction hydrotherapy as a component of complex physical therapy in disorders of the cervical and lumbar spine: a controlled pilot study with follow-up. Rheumatol Int. 2008 Jun;28(8):749-56. doi: 10.1007/ s00296-008-0522-y. Epub 2008 Jan 12. PubMed PMID: 18193231.

Disclosure of Interests: None declared

DOI: 10.1136/annrheumdis-2019-eular.4177

\section{THU0494 RELATIONSHIP BETWEEN THE AREA OF THE MEDIAN NERVE CROSS SECTION AND THE CIRCUMFERENCE OF THE CARPUS AS A DIAGNOSTIC TOOL FOR CARPAL TUNNEL SYNDROME}

Carlos Guillén-Astete, Patricia García-Casado. Hospital Ramón Y Cajal, MADRID, Spain

Background: The diagnosis of carpal tunnel syndrome (CTS) is fundamentally electrophysiological, however, on diagnostic suspicion ultrasonography has been shown to correlate satisfactorily with the electromyogram. The limitations of the ultrasound study of the median nerve in patients with STC are due to the fact that the intervals of normality of the area of the median nerve cross section (ACTNM) are variable according to the sources consulted, the sex and the anthropometry of the individual. Objectives: The purpose of this study is to determine whether NMTA can be correlated with a simple measurement such as wrist circumference length (MCL) in patients with electrophysiological diagnosis of TCEs and healthy subjects and used to discriminate them better than with the simple measurement of NMTA.

Methods: We included 50 patients with electrophysiological diagnosis of CTS and 43 healthy subjects of white ethnicity, older and with different anthropometric characteristics. The patients came from the Rheumatology consultations of three different centres. Healthy volunteers were subjects without CTS clinic, thyroid alterations, diabetes or known autoimmune rheumatological diseases whose data were obtained from a previous study. Circumference was measured with a flexible tape measure around the carpus immediately distal to the interstylloid line. The ultrasound measurements were made at the height of the escofoid and pisciforme bones using three different ultrasound scanners according to the headquarters: Toshiba Nemio XG, $13 \mathrm{Mhz}$ linear probe, Samsung HM70a 14 $\mathrm{MHz}$ and Logiq e GE $12 \mathrm{MHz}$. All the measured images were captured for analysis and correction of circumference lengths by an observer not linked to the identity of the subjects nor to their character of patient or control. An association study between wrist circumference and ACTNM was performed for both groups and a correlation index was submitted to a validation test for the determination of sensitivity and specificity.

Results: ACTNM was 11.11 SD $1.18 \mathrm{~mm} 2$ in the control group and 12.73 SD $1.50 \mathrm{~mm} 2$ in the patient group $(p<0.05)$. The LCM was $18.81 \mathrm{DE}$ $1.50 \mathrm{~cm}$ and $18.72 \mathrm{DE} 1.85(\mathrm{p}=0,803)$. In healthy subjects, the correlation between ACTNM and carpal circumference showed a satisfactory correlation (Coef Pearson 0.809, $\mathrm{p}<0.01$, bilateral) as well as in patients (Coef Pearson $0.878, \mathrm{p}<0.01$, bilateral). The ACTNM/LCM index in the control group was 0.590 and for the patient group $0.679(p<0.01)$. The area under the ACTNM curve was $0.808 \mathrm{EE} 0.049$ while in the case of the ACTNM/LCM index was $0.954 \mathrm{EE}$ 0.019. The area under the ACTNM curve was $0.808 \mathrm{EE} 0.049$ while in the case of the ACTNM/LCM index was $0.954 \mathrm{EE} 0.019$. With an index of 0.62 or a sensitivity of $93 \%$ and specificity of $78 \%$ are obtained for the diagnosis of CTS

Conclusion: LCM correlates well with ACTNM in healthy patients. It could be considered a good anthropometric marker for further studies of normal ultrasound ranges of the median nerve. The LCM/ACTNM index is a useful measure to discriminate controls of patients with electrophysiological diagnosis of JTS. In our series, this index exceeds the discriminatory capacity of the ACTNM as an individual measure.

Disclosure of Interests: : None declared

DOI: 10.1136/annrheumdis-2019-eular.8120

\section{THU0495 ROLE OF PLATELET RICH PLASMA IN TREATMENT OF ROTATOR CUFF TENDINOPATHY AND PARTIAL THICKNESS TEAR: FOLLOW UP BY ULTRASOUND}

Yasmin Khairy, Mona Nasr, Fatma Ali, Rasha Ali, Mohammed Abdelhakeem, Adham Khalil. Faculty of Medicine, Minia University, Egypt., Rheumatology and Rehabilitation Department, Minia, Egypt

Background: Shoulder pain is the third most common musculoskeletal reason for seeking medical care. The diagnosis of Rotator Cuff Tendinopathy (RCT), with supraspinatus partial thickness tendon tears and tendonosis, constitutes more than $50 \%$ of adult cases presenting with shoulder pains at any time. Platelet rich plasma (PRP) injections are nowadays being used as an alternative for treating the tendinopathies, who have failed to be managed by conservative management.

Objectives: This work aimed to asses the effect of PRP injection under musculoskeletal ultrasound (MSUS) guidance in patients with rotator cuff tendinopathy, and partial thickness tear in comparison with those who received a rehabilitation program only. Baseline assessment and after three months was done using clinical, functional and ultrasonographic evaluation.

Methods: Our study included 60 patients with RCT diagnosed both clinically and by MSUS. Patients were divided into two groups (gl, gll); group I included 30 patients who received a Supervised Rehabilitation Program and group II included 30 patients who received PRP injection. Patients in both groups were assessed clinically, functionally [(VAS) (WORC) and (SPADI)] and sonographically at baseline and after 3 months. Rehabilitation Program included: hot packs, (TENS), and (therapeutic ultrasound). The Exercise Programs (supervised and home-based) were applied, including: (ROM, stretching and strengthening exercises of the rotator cuff and scapular muscles). PRP injection was prepared under complete sterile conditions by whole blood centrifugation with specific 
protocol; blood was centrifuged firstly at $1000 \mathrm{rpm}$ for 10 minutes. The plasma was then transferred to a new glass tube and centrifuged at $3000 \mathrm{rpm}$ for 15 minutes. Platelets will form a pellet at the bottom of the tube. Finally, a pure platelet rich plasma was obtained with a concentration 4 times greater than baseline. Ca gluconate was mixed with PRP (in a ratio of $0.3 \mathrm{ml}$ ca gluconate $/ \mathrm{ml} \mathrm{PRP}$ ) immediately before the injection. Under ultrasound guidance, $3 \mathrm{ml}$ PRP was injected slowly into the bursa without usage of local anesthetics prior to injection. Post injection, patients were advised to rest, use cold packs and were allowed to do light range of motion exercises $2-5$ days post injection. Acetaminophen was allowed for intolerable post injection pain.

Results: Statistical analysis was made to 60 patients. Intragroup analysis showed statistical significant difference in both groups at follow up compared to baseline regarding clinical, functional and radiological data. Intergroup analysis showed more significant results in PRP group regarding clinical assessment ( $p<0.0001$ ), functional assessment (SPADI (PS, DS and total) and WORC scores $(p<0.0001)$ and sonographic assessment in (subscapularis tendinopathy, supraspinatus tendinopathy, supraspinatus fibrillar tendon disruption and supraspinatus tendon thickness) ( $p<0.0001)$ and sonographic subacromial subdeltoid bursitis $(p=0.001)$

Conclusion: Single PRP injection is an effective mean of treatment of RCT as it improves patients' quality of life clinically, functionally and structurally, better than traditional physical therapy program.

\section{REFERENCES:}

[1] Jacobson JA (2011). Shoulder US: anatomy, technique, and scanning pitfalls. Radiology; $260(1): 6-16$.

[2] Lansdown DA and Fortier LA (2017). Platelet-Rich Plasma: Formulations, Preparations, Constituents, and Their Effects. Operative Techniques in Sports Medicine; 25(1):7-12.

Disclosure of Interests: None declared

DOI: 10.1136/annrheumdis-2019-eular.4218

\section{THU0496 RESULTS OF AN EARLY INTERVENTION PROGRAM ON THE VARIATIONS IN THE LENGTH OF MUSCULOSKELETAL TEMPORARY WORK DISABILITIES}

Ana Lois-Iglesias ${ }^{1}$, Clara Ventin-Rodriguez ${ }^{1}$, Maria Caeiro-Aguado ${ }^{1}$, Alvaro SeijasLopez $^{1}$, Rodrigo Aquirre-del-Pino ${ }^{1}$, Francisco Javier de-Toro-Santos ${ }^{1,2} .1$ University Hospital A Coruña, A Coruña, Spain; ${ }^{2}$ University A Coruña, A Coruña, Spain

Background: Musculoskeletal disorders cause in Spain $23 \%$ of temporary work disability (TD). They are the first cause of permanent work disability (PD). A study of early intervention by a rheumatologist reduced TD days reduced PD. Using the "Fit for Work" European coalition led by AbbVie, the program was implemented nationwide.

Objectives: To analyze the variation in the duration of sick leaves in patients referred to an early intervention program in relation to the delay on the referral from primary care and from the Occupational Health service of our hospital.

Methods: Cross-sectional observational study of a hospital cohort of patients referred for 34 consecutive months to the Rheumatology Early Intervention program due to TDdue to musculoskeletal pathologies. Patients whoseTD had a traumatic or surgical origin, or with incomplete information were excluded from the analysis. We created 4 groups of patients according to the time elapsed from the start of the TD until the referral to our office: 0 to 15 days, 15 to 30 days, 30-60 days or $>90$ days. We compared the patients referred from primary care with those referred from occupational health.

Results: For the analysis we included 394 patients, 63.3\% women, with a mean age $( \pm D$.E. $)$ of $48.5( \pm 9.8)$ years. We analyzed the most frequent pathologies: back pain (33.5\%), shoulder pain (19.8\%) and neck pain (8.4\%). $85.8 \%$ came from primary care, $10.2 \%$ from occupational health and $4.1 \%$ from other units. The median time between referral and 1 st consultation was 6 days. We found statistically significant differences in the total duration of TD among the patients referred to consultation in the first 30 days compared to those sent later in the 3 pathologies (decreasing the duration of TD between 50 and 100 days in low back pain, between 44 and 54 days in shoulder pain and between 13 and 20 days in neck pain). In back pain we found statistically significant differences in the duration of the TD after the 1st consultation, obtaining an average decrease in the duration of the TD between 42 and 52 days. In shoulder pain and neck pain an important decrease is observed without reaching statistical significance, probably related to the sample size.
Regarding the patients referred from occupational health, the median time between TD and referral was 2.5 days. The pathology most frequently evaluated was low back pain $(60 \%$ of cases), finding statistically significant differences in the total duration of TD, with a mean decrease in the duration of TD of 59 days compared to the general population.

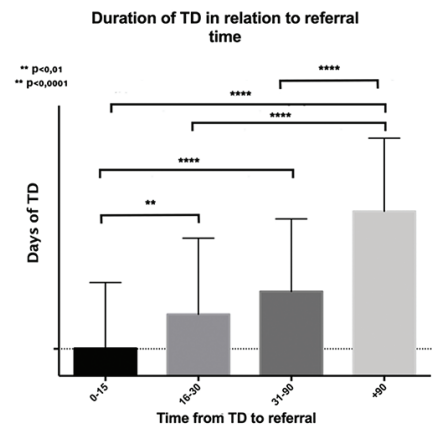

Abstract THU0496 - Figure 1

Conclusion: Early intervention by rheumatologists in patients with TD due to musculoskeletal disorders reduces the duration of the processes, saving costs to the health system.

Disclosure of Interests: None declared

DOI: 10.1136/annrheumdis-2019-eular.4388

\begin{tabular}{l|l}
\hline THU0497 & THE EFFECT OF ADDITION OF BUFFERED DEXTROSE \\
SOLUTION ON PAIN OCCURRING DURING LOCAL \\
STEROID INJECTION FOR PLANTAR FASCIITIS
\end{tabular}

Abd alhafez Moshrif ${ }^{1}$, Mohamed Elwan'2. ${ }^{1}$ Al Azhar University, Rheumatology, Assiut, Egypt, ${ }^{2}$ Al Azhar University, Rheumatology, Assiut, Egypt

Background: 5\%dextrose water (D5W) has been previously reported to decrease pain when co-administered with noxious agents as chemotherapeutics and microspheres ${ }^{(1,2)}$. It has also been reported to have an immediate analgesic effect on low back pain and radiculopathy when injected epidurally ${ }^{3}$.

Objectives: To evaluate the potential immediate analgesic effect of D5W when added to the injectate during local steroid injection for treatment of plantar fasciitis.

Methods: In this single blind study, a total of 122 patients with plantar fasciitis were randomly assigned to receive either $40 \mathrm{mg}$ triamcinolone acetonide $/ 1 \mathrm{ml}+0.5 \mathrm{ml}$. lidocaine $2 \%$ (group A: 61 patients; 73 heels) or $40 \mathrm{mg}$ triamcinolone acetonide $/ 1 \mathrm{ml}+0.5 \mathrm{ml}$. lidocaine $2 \%+0.5 \mathrm{ml}$. buffered D5W (group B: 61 patients; 69 heels) as a local injection using the medial approach. Clinical assessment including disease duration, BMI, history of previous injection and post-injection complications and 2 week recurrence rate was performed. Plain X-ray lateral view on the painfu heel was obtained for diagnosis of associating calcaneal spur. Visual analogue scale (VAS 0-10) was used to assess the degree of pain intensity during injection.

Results: There were no significant difference between both groups regarding age, sex or BMI where the mean for age was 42.56 years in group $A$ and 43.39 years in group $B(P=0.86)$, the male to female ratio was 16:45 in both groups and the mean for BMI was 31.49 in group $A$ and 30.86 in group $B(P=0.51)$. The mean disease duration was 6.02 months in group $A$ and 10.77 months in group $B(P=0.005)$. Calcaneal spur was diagnosed in 60 patients (82\%) in group $A$ and in 47 patients (68\%) in group $B$. A highly significant difference in VAS was observed as the mean was $8.26 \pm 2.00$ in group $A$ and $4.25 \pm 2.05$ in group $B$ ( $P$ $<0.0001)$ with a confidence interval $(95 \% \mathrm{Cl})$ of $7.78-8.74$ for group $\mathrm{A}$ and $3.76-4.72$ for group B. On 2 week follow up, only 4 patients in each group reported a recurrent heel pain. No injection related side effects or complications have been reported.

Conclusion: The addition of $0.5 \mathrm{ml}$. D5W can significantly decrease the pain associated with local steroid injection for treatment of plantar fasciitis.

\section{REFERENCES:}

[1] T. Nakashima, Y. Ogawa, A. Kimura et al., "Coadministration of $5 \%$ glucose solution has a decrease in bendamustine-related vascular pain grade," Journal of Oncolog y Phar mac y Prac tice, vol. 18, no. 4, pp 445-447, 2012. 